\title{
Réponses analytiques aux intoxications par substances végétales
}

\section{Analytical answer to poisoning by plant material}

Marjorie CHEZE, Yvan GAILLARD, Gilbert PÉPIN

Laboratoire d'Expertises TOXLAB, 18 rue André Del Sarte - 75018 PARIS

Tél : 0142620030 - Fax : 0142573959 - e-mail : labtoxlab@aol.com

\section{$R E S U M E ́$}

Environ une quarantaine de plantes (genre ou espèce) sont responsables de $95 \%$ des décès publiés dans la littérature lors d'intoxication par les plantes. Bien que la plupart de ces toxiques soient mal connus, les molécules actives en cause peuvent être détectées par des méthodes analytiques telles que la chromatographie en phase gazeuse ou la chromatographie en phase liquide. Les méthodes analytiques publiées ne sont pas nombreuses et souvent peu sensibles ou peu spécifiques ou encore dédiées à quelques molécules ou métabolites. Nous avons développé une méthode générale pour la détection des principaux toxiques dans le sang total par chromatographie liquide couplée à la spectrométrie de masse simple ou tandem. L'extraction des molécules en liquide-liquide est conduite à pH 9,5 pour l'oléandrine, le paclitaxel et certains alcaloides. Les alcaloïdes bases faibles nécessitent une purification acide /base supplémentaire. L'Atractyloside est isolé par précipitation des protéines à l'acétone et lavage par une phase organique. La séparation chromatographique s'effectue en phase inverse sur colonne C18 $150 \times 2 \mathrm{~mm}$ i.d. , $5 \mu \mathrm{m}$ d.p. au moyen de deux phases mobiles à différents $\mathrm{pH}$. Un tampon formiate d'ammonium $2 m M$ acidifié à $p H 3$ est utilisé pour la séparation de l'atractyloside, de l'oléandrine et du paclitaxel et certains alcaloïdes. Un tampon formiate $10 \mathrm{mM}$ à pH 8,2 est utilisé pour la majorité des autres alcaloïdes. Le gradient d'élution utilise l'acétonitrile. La détection en mode positif est le mode de choix pour la majorité des alcaloïdes excepté pour l'atractyloside (mode négatif) et le paclitaxel (deux modes possibles). L'application à des cas médico-légaux est présentée ici.

\section{MOTS-CLÉS}

Plantes, Intoxications, Chromatographie liquide, Spectrométrie de masse, Spectrométrie de masse tandem, Alcaloüdes, Atractyloside, Oléandrine, Paclitaxel, Toxicologie médico-légale.
(Reçu le 10 octobre 2000 ; accepté le 30 octobre 2000)

\section{SUMMARY}

About forty plants (genera or species) are responsible for 95\% of the published fatal cases due to plant poisoning. Although many available toxins are not well known the reviewed active molecules can be identified by analytical methods like gas chromatography or liquid chromatography. The published analytical methods are not numerous and not always sensitive or specific or are dedicated just to some molecules or metabolites. We have developed a general method for the detection of main toxins in whole blood by high performance liquid chromatography - mass spectrometry or tandem mass spectrometry. Sample preparation was realized by liquid-liquid extraction at $\mathrm{pH}$ 9,5 for oleandrine, paclitaxel and some alkaloids. The extract of weak base alkaloids must be purified by acid/base clean up. Atractyloside was isolated by precipitation of the protein content with acetone and purified for atractyloside by washing with organic solvent. Separation of the drugs occurred under reversed phase conditions on a C18 analytical

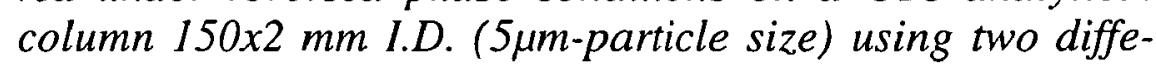
rent $\mathrm{pH}$ mobile phases. Formiate buffer $2 \mathrm{mM}$ acidified at $\mathrm{pH}$ 3,0, was used for the separation of atractyloside, oleandrine and paclitaxel and some alkaloids. Formiate buffer $10 \mathrm{mM}$ made basic at $\mathrm{pH} 8.2$ was used for the majority of others alkaloids. A gradient elution mode was chosen using acetonitrile as the eluting solvent. Detection under positive ionisation mode was the preferred mode for all compounds except for atractyloside (negative ions) and for taxol (mixed mode available). Application to real forensic cases has been demonstrated and is presented here.

\section{KEY-WORDS}

Plants, Intoxications, Liquid chromatography, Mass spectrometry, Tandem Mass spectrometry, Alkaloids, Atractyloside, Oleandrine, Paclitaxel, Forensic toxicology. 


\section{Introduction}

Des études rétrospectives sur ces vingt dernières années ont estimé l'incidence de l'empoisonnement aux végétaux à environ $1,5 \%$ en France, $5 \%$ en Belgique, $6,5 \%$ en Italie, $7,2 \%$ en Suisse et $6 \%$ en Turquie (1). Aux Etats Unis, entre 1985 et 1994, ces empoisonnements se situaient en quatrième position après les intoxications aux médicaments et drogues, les produits ménagers et les cosmétiques (2).

Cependant, le nombre des décès reste difficile à établir et est probablement sous estimé car peu documenté par des travaux analytiques, encore délicats, et qui plus est médico-légaux $(3,4)$. Plus particulièrement, nous avons eu à traiter à Paris ces dernières années plusieurs cas d'intoxications ou d'empoisonnements par l'utilisation de plantes connues en médecines traditionnelles. A la demande de la commission médico-légale de la S.F.T.A. (Société Française de Toxicologie Analytique) nous avons travaillé sur la mise au point d'une technique analytique de screening des principales plantes en cause dont les 22 principaux toxiques abordés ici sont listés dans le Tableau I $(5,6)$.

\section{Matériel et méthode}

\section{Réactifs}

Le méthanol, l'acétone, le dichlorométhane et le chloroforme sont de qualité HPLC (Prolabo), l'acétonitrile, l'isopropanol, l'acide formique, le formiate d'ammonium, l'ammoniaque ( $30 \%$ ), le chlorure d'ammonium, l'acide chlorhydrique $0.5 \mathrm{~N}$ et la soude $1 \mathrm{~N}$ sont de qualité pour analyse (Carlo Erba). L'extraction liquideliquide utilise une solution tampon de $\mathrm{NH} 4 \mathrm{Cl}$ saturée ajustée à pH 9,5 par l'ammoniaque. Le tampon formiate d'ammonium $2 \mathrm{mM}$ ajusté à $\mathrm{pH} 3$ par l'acide formique est utilisé pour la séparation chromatographique des alcaloïdes de $\mathrm{pK}<6,5$. Le tampon formiate $10 \mathrm{mM}$ ajusté à $\mathrm{pH} 8,2$ par l'ammoniaque est utilisé pour la séparation des alcaloïdes de $\mathrm{pK}>6,5$.

Les solutions standard d'alcaloïdes sont réalisées à 10 , 1 et $0,1 \mu \mathrm{g} / \mathrm{ml}$ dans le méthanol à partir d'étalons disponibles chez Extrasynthèse (Genay, France) ou Sigma (Saint Quentin Fallavier, France).

\section{Extraction}

Les alcaloïdes ont été divisés en deux groupes selon leurs propriétés physico-chimiques. Les alcaloïdes

Tableau I : Les 22 principaux alcalö̈des toxiques végétaux connus (hors stupéfiants, hallucinogènes...).

\begin{tabular}{|c|c|c|}
\hline Alcaloide Toxique Majoritaire & Nom Eatin & Nom Commun \\
\hline aconitine & Genus Aconitum & Aconit \\
\hline atropine & Atropa Belladona & Belladone \\
\hline scopolamine & Hyoscyamus Niger & Hyoscyamus \\
\hline scopolamine, atropine & Datura Stramonium & Datura \\
\hline vératrine (cévadine, vératridine...) & Veratrum Viride & Vératre \\
\hline colchicine & Colchicum Automnale & Colchique \\
\hline réserpine & Rauwolfa Serpentina & Sarpagandha de l'Inde \\
\hline sénécionine & Senecio Vulgaris & 1 \\
\hline strychnine & Strychnos nux vomica & Vomiquier (noix vomique) \\
\hline arécoline & Areca Catechu & Noix de Bétel ou d'Arec \\
\hline émétine & Uragoga Ipecacuanha & Ipéca (racines) \\
\hline ésérine & Physostigma Venenosum & Fève de Calabar \\
\hline oléandrine & Nerium Oleander & Laurier rose \\
\hline rétrorsine & Senecio Retrorsus & 1 \\
\hline spartéine (lupinidine) & Cytisus Scoparius & Genêt à Balais \\
\hline paclitaxel (taxine A, taxol) & Taxus & If \\
\hline yohimbine & Pausinystalian Yohimbe & Yohimbe \\
\hline anabasine (néonicotine) & Anabasis aphylla/Nicotina Glauca & 1 \\
\hline crotaline & Crotalaria & 1 \\
\hline lobéline & Lobelia Inflata & Lobélie \\
\hline pilocarpine & Pilocarpus Microphyllus & Jaborandi de Maranham \\
\hline atractyloside & Atractylis Glummifera & Chardon à glu \\
\hline
\end{tabular}


appelés groupe A : anabasine, arécoline, atropine, crotaline, émétine, ésérine, lobeline, pilocarpine, rétrorsine, scopolamine, sénéscionine, sparteine, yohimbine, de "faible" poids moléculaire, sont extraits par une triple extraction en phase liquide afin d'obtenir un extrait le plus propre. $2 \mathrm{ml}$ d'échantillon sont tamponnés à $\mathrm{pH} 9,5$ par $2 \mathrm{ml}$ de la solution de $\mathrm{NH} 4 \mathrm{Cl}$ saturée puis extraits par $10 \mathrm{ml}$ d'un mélange chloroforme / isopropanol $(95 / 1, v / v)$. La phase organique est réextraite à $\mathrm{pH}$ acide par $5 \mathrm{ml}$ de $\mathrm{HCl} 0,5 \mathrm{~N}$ puis écartée. La phase aqueuse est neutralisée par 2,5 ml de $\mathrm{NaOH} 1 \mathrm{~N}$, alcalinisée à $\mathrm{pH} 9,5$ par $4 \mathrm{ml}$ de solution $\mathrm{NH} 4 \mathrm{Cl}$ puis réextraite par $10 \mathrm{ml}$ de chloroforme ou dichlorométhane. L'extrait est évaporé à sec sur bloc chauffant sous courant d'air et repris par $30 \mu \mathrm{l}$ d'acétonitrile pour l'injection. Les alcaloïdes du groupe B: aconitine, colchicine, cévadine, oléandrine, réserpine, strychnine, taxol et vératridine, de plus haut poids moléculaire, peuvent être extraits par simple extraction liquide-liquide, et reprise directe de l'extrait pour injection. Une triple extraction diminue le rendement sans améliorer le rapport signal sur bruit.

L'atractyloside est isolé par précipitation des protéines à l'acétone et par un lavage de l'extrait au chloroforme ou au dichlorométhane. La phase aqueuse est évaporée et le résidu sec repris par $40 \mu \mathrm{l}$ d'un mélange acétonitrile / eau $(50 / 50, \mathrm{v} / \mathrm{v})$ pour injection.

\section{Instrumentation}

L'analyse de routine est réalisée par chromatographie liquide couplée à la spectrométrie de masse. Le système chromatographique comporte un injecteur automatique AS 200 (Perkin Elmer) et deux micro pompes serie 200 couplées à un spectromètre de masse API Sciex 165 muni d'un interface électrospray assisté pneumatiquement.

Pour une meilleure sensibilité et spécificité la chromatographie liquide couplée à la spectrométrie de masse tandem est une excellente alternative. Le système est constitué d'une pompe quaternaire P4000 et d'un injecteur automatique AS 100XR de chez TSP (Thermo Separation Products). Le spectromètre de masse est un TSQ 7000 de chez Thermoquest muni d'un interface électrospray à capillaire d'introduction chauffé.

\section{Séparation chromatographique}

Les séparations chromatographiques sont réalisées sur colonne C18-ODS - $150 \times 2 \mathrm{~mm}$ - $5 \mu \mathrm{m}$ (InterchimUptisphère) munie d'une pré-colonne de $10 \mathrm{~mm}$ possédant les mêmes caractéristiques.

Les alcaloïdes du groupe $\mathrm{A}$, de pK supérieur à 6,5, peu hydroxylés et de "faible" poids moléculaire sont séparés dans des conditions basiques à $\mathrm{pH} 8,2$ avec un gradient de phase de $80 \%$ de tampon et $20 \%$ d'acétonitrile/méthanol $(2 / 1, v / v)$ aux conditions initiales pendant une minute puis linéairement diminué à $10 \%$ de tampon au bout de 10 minutes et maintenu 12 minutes. La colonne est rééquilibrée 15 minutes aux conditions initiales.

Les alcaloïdes du groupe $\mathrm{B}$, de $\mathrm{pK}$ inférieur à 6,5, hydroxylés et de haut poids moléculaire sont séparés dans des conditions acides à $\mathrm{pH} 3$ avec un gradient de phase de $80 \%$ de tampon et $20 \%$ d'acétonitrile aux conditions initiales pendant une minute puis linéairement diminué à $10 \%$ de tampon au bout de 20 minutes et maintenu 5 minutes. La colonne est rééquilibrée 15 minutes aux conditions initiales. Le débit est de 250 $\mu 1 /$ min auquel est appliqué un split de $1 / 3$ en sortie de colonne pour optimiser le débit dans l'interface.

Ces conditions chromatographiques utilisées en première intention ont l'inconvénient d'amener à une dégradation rapide de la colonne $\mathrm{C} 18$ classique si celleci n'est pas rincée rapidement après l'utilisation du tampon $\mathrm{pH} 8,2$. Un temps important de rééquilibration de la colonne entre ces deux phase mobiles est nécessaire pour obtenir des résultats répétables. Des essais ont été effectués sur une colonne de dernière génération XTerra MS C18 Waters $(150 \times 2,1 \mathrm{~mm}-3,5 \mu \mathrm{m})$ résistant à de larges gammes de $\mathrm{pH}$. Une réduction notable des temps de rétention permettant d'écourter l'analyse a été observée et la stabilité aux changements de phases permet une plus grande souplesse d'utilisation. Les limites de détection ne semblent pas notablement modifiées (figures 1 et 2 ).

\section{Détection}

La majorité des alcaloïdes est détectée en mode positif avec une tension d'orifice de $+60 \mathrm{~V}$ et une tension de spray de $+5,2 \mathrm{kV}$. Le mode négatif est utilisé pour la détection du paclitaxel (alternative au mode positif) et pour l'atractyloside (sel dipotassique d'un acide organique) avec une tension d'orifice de $-60 \mathrm{~V}$ et une tension de spray de $-4,2 \mathrm{kV}$.

\section{Résultats}

Les limites de détection obtenues applicables à la résolution de cas médicaux légaux sont présentés dans le tableau II. La répétabilité était satisfaisante avec un coefficient de variation inférieur à 16,2\%. La reproductibilité sur l'ensemble des alcaloïdes $(n=10)$ à 100 $\mathrm{ng} / \mathrm{ml}$ montrait un coefficient de variation inférieur à $11,2 \%$ (excepté pour la lobéline $=16,2 \%$ ), à $10 \mathrm{ng} / \mathrm{ml}$ un coefficient de variation inférieur à 16,0\% (excepté pour la lobéline $=21.8 \%$ ), à $1 \mathrm{ng} / \mathrm{ml}$ un coefficient de variation inférieur à 19,0\% (tableau III). Les rendements d'extraction sont indiqués dans le tableau IV (7). 


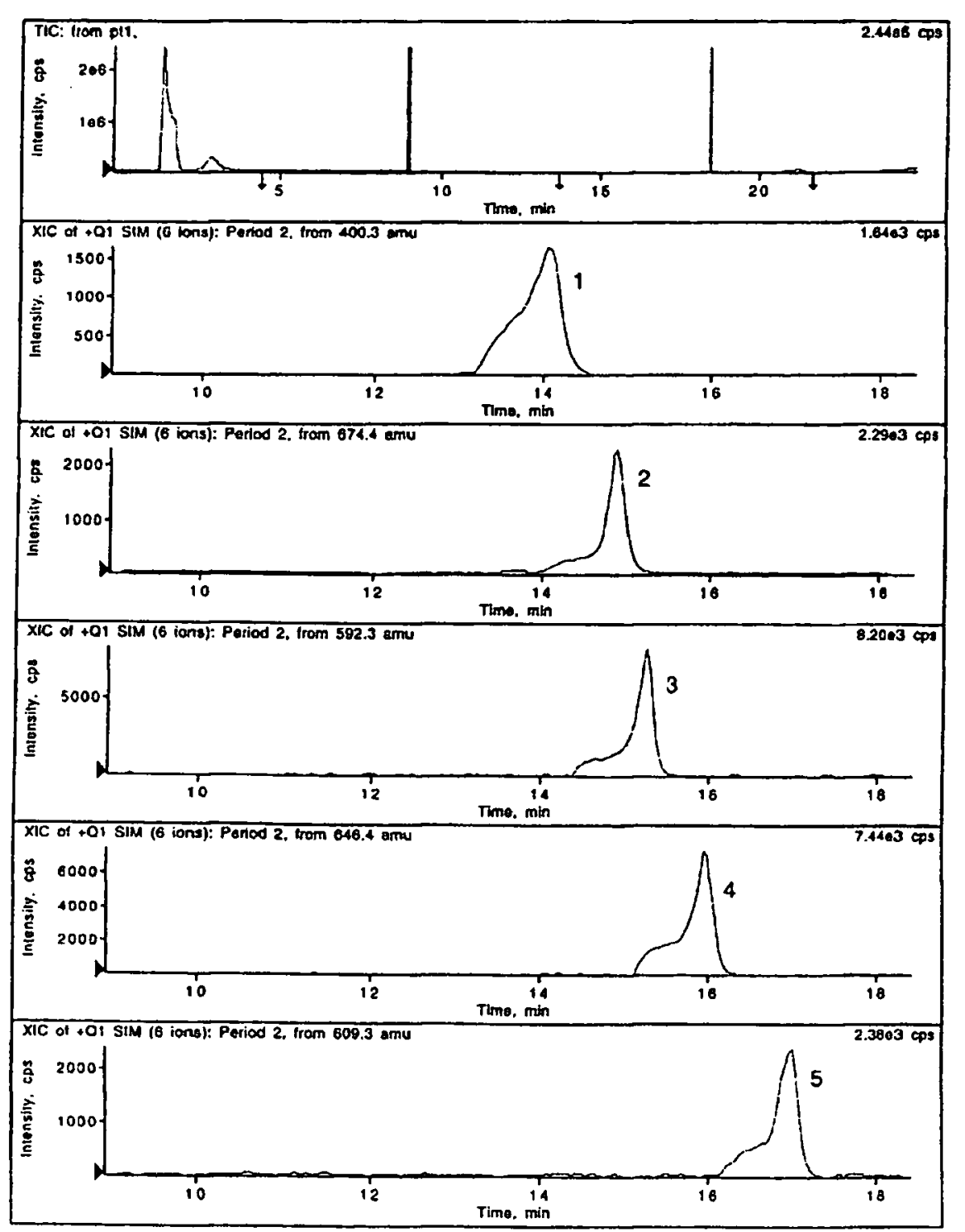

Figure 1 : Chromatogramme sur colonne ODS d'un extrait de sang total témoin avec ajout de $1 \mathrm{ng} / \mathrm{ml}$ de chaque molécule. Pics : 1 = colchicine $(\mathrm{m} / \mathrm{z} 400,3), 2$ = vératridine $(\mathrm{m} / \mathrm{z} 674,4), 3=$ cévadine $(\mathrm{m} / \mathrm{z} 592,3), 4=$ aconitine $(\mathrm{m} / \mathrm{z} 646,4), 5=$ réserpine $(\mathrm{m} / z$ 609,3).

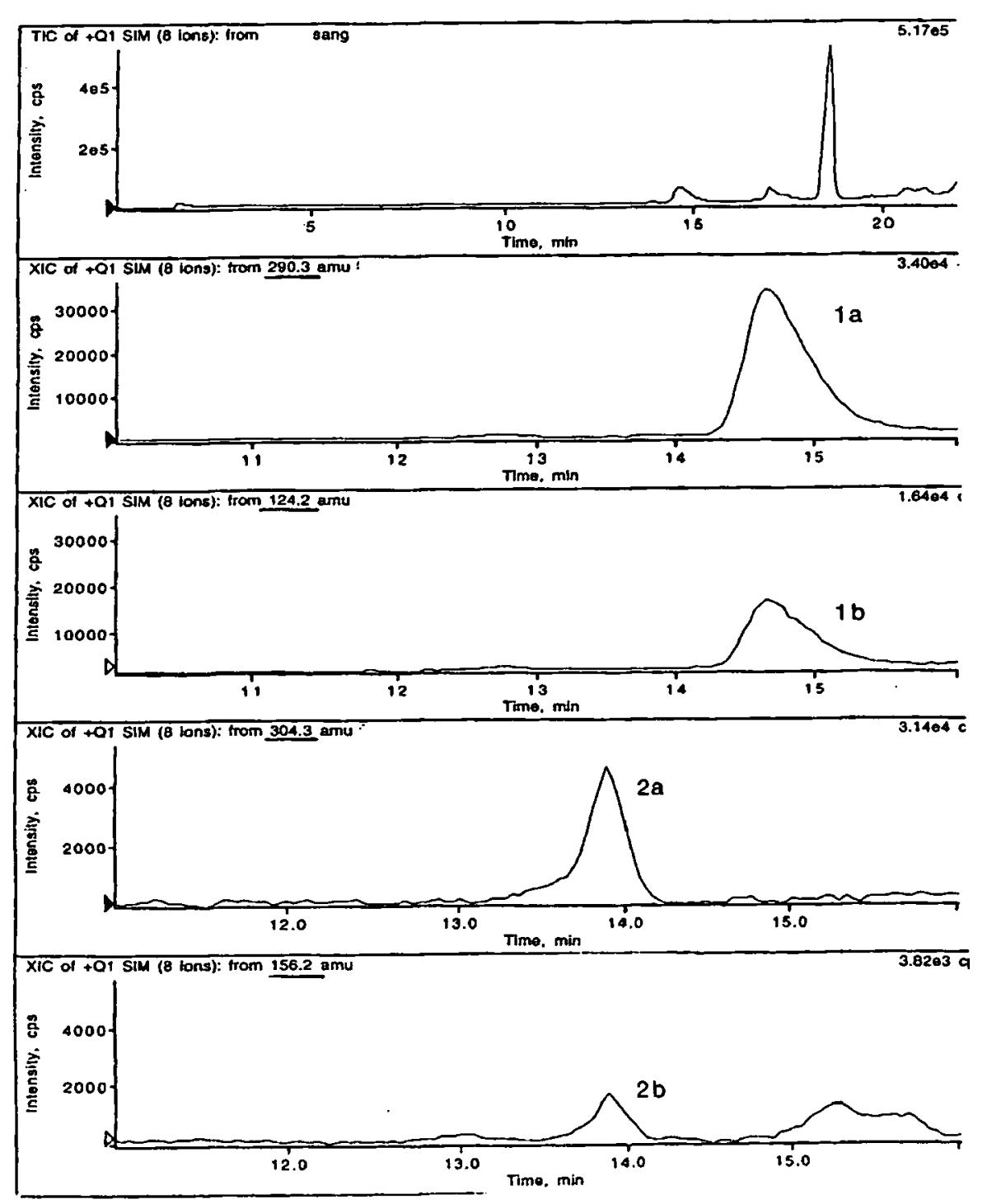

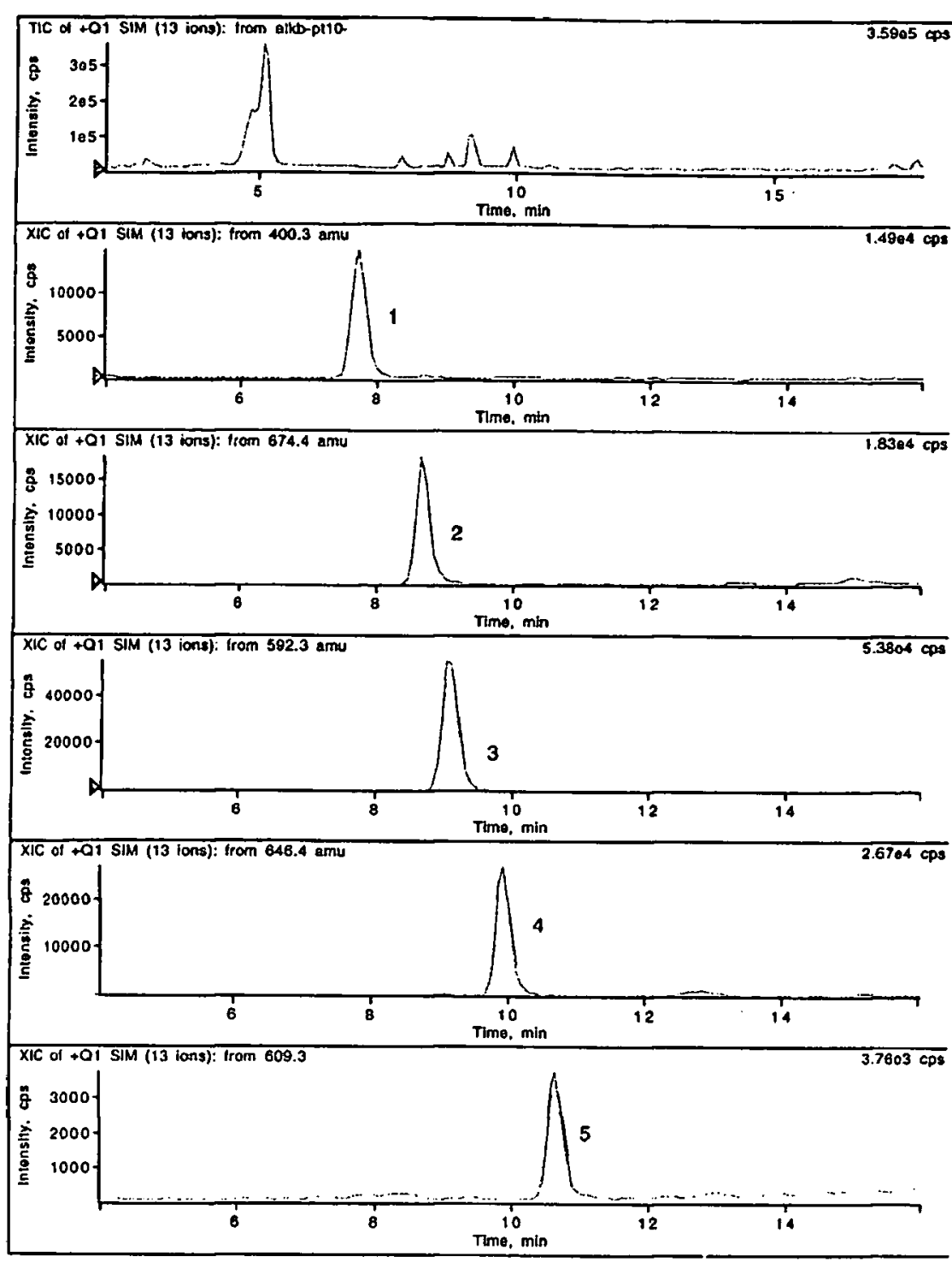

Figure 2 : Chromatogramme sur colonne XTerra d'un extrait de sang total témoin avec ajout de $10 \mathrm{ng} / \mathrm{ml}$ de chaque molécule. Pics : $1=$ colchicine $(\mathrm{m} / \mathrm{z} 400,3), 2=$ vératridine $(\mathrm{m} / \mathrm{z} 674,4), 3=$ cévadine $(\mathrm{m} / \mathrm{z} 592,3), 4=$ aconitine $(\mathrm{m} / \mathrm{z} 646,4), 5=$ réserpine $(\mathrm{m} / \mathrm{z} 609,3)$.

Tableau II : Limites de détection en LC/ESI-SM sur colonne ODS $150 \times 2 \mathrm{~mm}$.

\begin{tabular}{|c|l|}
\hline $\begin{array}{c}\text { Limites de } \\
\text { Détection en ng/ml }\end{array}$ & \multicolumn{1}{c|}{ Alcaloïdes } \\
\hline$<0,1$ & $\begin{array}{l}\text { aconitine, atropine, cévadine, } \\
\text { colchicine, réserpine, sénécionine, } \\
\text { strychnine, vératridine }\end{array}$ \\
\hline $0,1-1$ & $\begin{array}{l}\text { arécoline, émétine, ésérine, } \\
\text { oléandrine, rétrorscine, scopolamine, } \\
\text { spartéine, paclitaxel, yohimbine }\end{array}$ \\
\hline$>1$ & $\begin{array}{l}\text { anabasine, crotaline, lobéline, } \\
\text { pilocarpine }\end{array}$ \\
\hline
\end{tabular}

Figure 3 ci-contre : Chromatogramme sur colonne ODS d'un extrait de sang total. Pics: $:$ la et $1 \mathrm{l} b=$ atropine $(\mathrm{m} / \mathrm{z}$ $290,3$ et 124,2$), 2 a$ et $2 b=$ scopolamine $(\mathrm{m} / \mathrm{z} 304,3$ et $156,2)$. 
Tableau III : Étude de reproductibilité, rendements d'extraction et LD des alcaloüdes, du paclitaxel et de l'oléandrine ( $n=10)$.

\begin{tabular}{|c|c|c|c|c|c|c|}
\hline Composé & $\begin{array}{l}\text { CV (\%) à } \\
0,1 \text { ng/ml }\end{array}$ & $\begin{array}{c}\mathrm{CV}(\%) \text { à } \\
1 \mathrm{ng} / \mathrm{ml}\end{array}$ & $\begin{array}{l}\text { CV (\%) à } \\
10 \mathrm{ng} / \mathrm{ml}\end{array}$ & $\begin{array}{l}\text { CV (\%) à } \\
100 \mathrm{ng} / \mathrm{ml}\end{array}$ & $\begin{array}{c}\text { Rendement } \\
\text { d'extraction } \\
\text { (\%) }\end{array}$ & $\begin{array}{c}\mathbf{L D}(\mathbf{n g} / \mathbf{m l}) \\
\mathbf{n}=\mathbf{3 0}\end{array}$ \\
\hline Aconitine & 8,8 & 6,1 & 4,8 & 4,2 & 86,4 & $0,01\left(0.001^{a}\right)$ \\
\hline Anabasine & - & - & 11,6 & 8,7 & 49,9 & 1,20 \\
\hline Arécoline & - & 19,0 & 8,0 & 5,6 & 63,1 & 0,91 \\
\hline Atropine & 11,8 & 6,1 & 4,2 & 3,7 & 70,9 & 0,09 \\
\hline Cévadine & 9,1 & 7,9 & 7,0 & 5,3 & 87,1 & $0,01\left(0.001^{a}\right)$ \\
\hline Colchicine & 24,6 & 13,4 & 9,9 & 8,2 & 51,1 & $0,09\left(0.005^{a}\right)$ \\
\hline Crotaline & - & - & 15,3 & 10,4 & 49,6 & 1,60 \\
\hline Emétine & - & 21,0 & 16,0 & 11,2 & 21,8 & 0,19 \\
\hline Esérine & - & 17,2 & 8,7 & 8,0 & 103,7 & 0,17 \\
\hline Lobéline & - & - & 21,8 & 16,2 & 18,3 & 1,52 \\
\hline Oléandrine & - & 14,0 & 8,7 & 7,3 & 53,5 & 0,40 \\
\hline Pilocarpine & - & - & 7,6 & 6,6 & 68,1 & 1,07 \\
\hline Réserpine & 11,7 & 9,5 & 8,8 & 8,1 & 50,3 & 0,02 \\
\hline Rétrorscine & - & 13,0 & 8,4 & 5,2 & 61,6 & 0,18 \\
\hline Scopolamine & - & 12,4 & 9,1 & 6,9 & 64,4 & 0,31 \\
\hline Sénécionine & 9,3 & 7,4 & 6,7 & 5,9 & 101,0 & 0,04 \\
\hline Spartéine & - & 8,4 & 7,2 & 6,0 & 63,3 & $0,16\left(0.005^{\mathrm{a}}\right)$ \\
\hline Strychnine & 10,9 & 9,4 & 6,1 & 5,4 & 100,2 & $0,03\left(0.002^{a}\right)$ \\
\hline Paclitaxel & - & 12,7 & 7,8 & 6,6 & 43,2 & 0,48 \\
\hline Vératridine & 11,7 & 9,2 & 6,3 & 4,1 & 86,6 & $0,02\left(0.001^{\mathrm{a}}\right)$ \\
\hline Yohimbine & - & 10,1 & 6,9 & 5,2 & 100,2 & 0,32 \\
\hline
\end{tabular}

- : Non déterminé

a : Analyse réalisée par CLHP-SM-SM

comme endormies, sans signe de tentative de fuite. L'enquête a finalement déterminé l'incendie comme étant d'origine criminelle et a permis de trouver au domicile familial des ouvrages sur les plantes toxiques. L'autopsie montrait des signes de violences ainsi que des résidus de fumées dans les trachées. Des prélèvements de sang, de cheveux et de contenu gastrique étaient effectués. Une analyse de première intention révélait la présence de toxiques dus à l'incendie ( $\mathrm{HbCO}$, cyanure) et à un traitement anti-épileptique (acide valproïque, clonazépam, carbamazépine) qui n'expliquaient pas à eux seuls l'absence de réaction des fillettes face au feu. Des analyses complémentaires étaient donc réalisées pour la recherche des toxiques végétaux (tableau $\mathrm{V}$ ). Le screening des alcaloïdes révélait la présence d'atropine et de scopolamine dans le sang (figure 3) et le contenu gastrique à des taux non mortels, cependant l'effet narcotique du Datura (hallucinogène narcotique incapacitant) les avait probablement laissées dans un état de stupeur semi-comateux pouvant expliquer leur incapacité de fuir $(8,9)$. Les parents ont finalement avoué avoir drogué leurs enfants avec des extraits de feuilles de Datura, fréquente dans cette région, puis les avoir tuées par le feu après que leur requête d'admission de celles-ci dans une institution spécialisée fut refusée. 
Tableau V : Cas 1 - Résultats d'analyse toxicologique.

\begin{tabular}{|c|c|c|c|}
\hline \multicolumn{2}{|l|}{ Fillette 1} & \multicolumn{2}{|l|}{ Fillette 2} \\
\hline sang & $\begin{array}{c}\text { carboxyhémoglobine }(\mathrm{HbCO})=11,3 \% \\
\text { acide valproique }=14,4 \mu \mathrm{g} / \mathrm{ml} \\
\text { clonazépam }=0,9 \mu \mathrm{g} / \mathrm{ml} \\
\text { cyanure }=2,4 \mu \mathrm{g} / \mathrm{ml} \\
\\
\text { atropine }=\mathbf{3 2 , 5} \mathbf{~ n g} / \mathrm{ml} \\
\text { scopolamine }=\mathbf{4 , 4} \mathbf{n g} / \mathrm{ml}\end{array}$ & sang & $\begin{array}{c}\mathrm{HbCO}=11,9 \% \\
\text { acide valproique }=8,3 \mu \mathrm{g} / \mathrm{ml} \\
\text { carbamazépine }=1,9 \mu \mathrm{g} / \mathrm{ml} \\
\text { cyanure }=0,7 \mu \mathrm{g} / \mathrm{ml} \\
\\
\text { atropine }=\mathbf{7 , 5} \mathbf{n g} / \mathrm{ml} \\
\text { scopolamine }=\mathbf{0 , 8} \mathbf{n g} / \mathrm{ml}\end{array}$ \\
\hline $\begin{array}{l}\text { contenu } \\
\text { gastrique }\end{array}$ & $\begin{array}{c}\text { atropine }=22,0 \mathrm{ng} / \mathrm{ml} \\
\text { scopolamine }=2,1 \mathrm{ng} / \mathrm{ml}\end{array}$ & $\begin{array}{l}\text { contenu } \\
\text { gastrique }\end{array}$ & $\begin{array}{c}\text { atropine }=18,3 \mathrm{ng} / \mathrm{ml} \\
\text { scopolamine }=1,1 \mathrm{ng} / \mathrm{ml}\end{array}$ \\
\hline
\end{tabular}

\section{CAS n ${ }^{\circ} 2$ : Vératre (Vératrine)}

Deux hommes blanc âgés d'une trentaine d'années furent repêchés dans un lac de montagne un mois après leur décès estimé. L'enquête révèlait, qu'immigrants clandestins d'origine européenne, ils vivaient dans une caravane dans laquelle était découverte un pot contenant quantité de graines sauvages d'environ $3 \mathrm{~mm}$ de diamètre. L'autopsie ne révélait aucune trace de traumatisme et la thèse d'un décès par noyade semblait peu probable de par l'absence d'eau et de débris significatifs dans les bronches et les poumons. Les contenus gastriques étaient roses avec de nombreuses graines noires semblables à celles retrouvées dans la caravane. Ces graines ainsi que du contenu gastrique et du sang étaient prélevés pour analyse toxicologique. Les résultats obtenus ont été regroupés dans le tableau VI. Les taux en strontium de l'eau du lac étaient très élevés comparés aux taux déterminés dans le sang des deux

Tableau VI : Cas 2 - Résultats d'analyse toxicologique.

\begin{tabular}{|c|c|c|}
\hline \multirow{2}{*}{ HI } & sang & $\begin{array}{c}\mathrm{Sr}=62 \mu \mathrm{g} / \mathrm{l} \\
\text { Cévadine }=0,32 \mathrm{ng} / \mathrm{ml} \\
\text { Vératridine }=0,17 \mathrm{ng} / \mathrm{ml}\end{array}$ \\
\hline & contenu gastrique & $\begin{array}{l}\text { Cévadine }=0,39 \mathrm{ng} / \mathrm{ml} \\
\text { Vératridine }=0,23 \mathrm{ng} / \mathrm{ml}\end{array}$ \\
\hline \multirow{2}{*}{$\mathrm{H} 2$} & sang & $\begin{array}{c}\mathrm{Sr}=86 \mu \mathrm{g} / \mathrm{l} \\
\text { Cévadine }=0,48 \mathrm{ng} / \mathrm{ml} \\
\text { Vératridine }=0,40 \mathrm{ng} / \mathrm{ml}\end{array}$ \\
\hline & contenu gastrique & $\begin{array}{l}\text { Cévadine }=0,71 \mathrm{ng} / \mathrm{ml} \\
\text { Vératridine }=0,50 \mathrm{ng} / \mathrm{ml}\end{array}$ \\
\hline Eau du lac & & $\mathrm{Sr}=458 \mu \mathrm{g} / 1$ \\
\hline \multirow{3}{*}{$\begin{array}{c}\text { Graines } \\
\text { (n) }\end{array}$} & caravane & $\begin{array}{c}\text { Cévadine }=0,23 \mathrm{mg} / \mathrm{g} \\
\text { Vératridine }=1,26 \mathrm{mg} / \mathrm{g}\end{array}$ \\
\hline & HI (contenu gastrique) & $\begin{array}{l}\text { Cévadine }=0,82 \mathrm{ng} / \mathrm{g} \\
\text { Vératridine }=0,38 \mathrm{ng} / \mathrm{g}\end{array}$ \\
\hline & H2 (contenu gastrique) & $\begin{array}{l}\text { Cévadine }=1,52 \mathrm{ng} / \mathrm{g} \\
\text { Vératridine }=1,0 \mathrm{ng} / \mathrm{g}\end{array}$ \\
\hline
\end{tabular}

victimes, confirmant l'absence de noyade. La présence de vératridine et de cévadine dans le bol alimentaire (figures 4 et 5) représentait la preuve de l'ingestion de Vératre, plante très toxique, dont l'analyse des graines corroborait les résultats. Malheureusement il n'existe pas de concentration post-mortem dans la littérature pour des raisons de difficultés analytiques de dosage . La dose létale est d'environ 1 à 2 grammes de poudre de plante sèche. Les alcaloödes du vératre exercent leur toxicité au niveau du muscle cardiaque. L'ensemble de ces éléments était en faveur d'une intoxication au Vératre. Les circonstances de l'ingestion comme celles de l'immersion restent indéterminées.
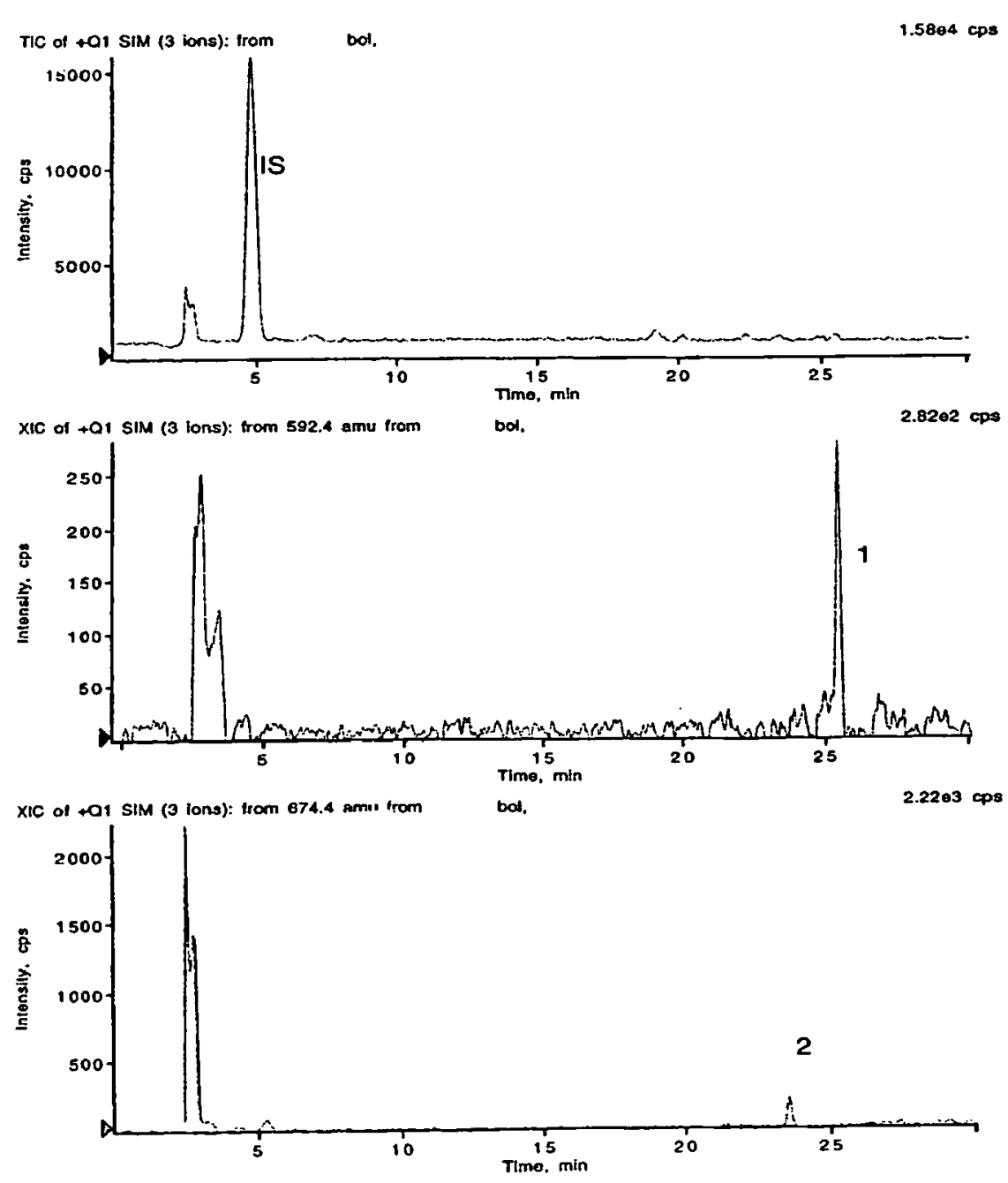

Figure $4:$ Chromatogramme sur colonne ODS d'un extrait de bol alimentaire. Pics : $1=$ cévadine $(\mathrm{m} / \mathrm{z} 592,4), 2=$ vératridine $(\mathrm{m} / \mathrm{z} 674,4)$. 

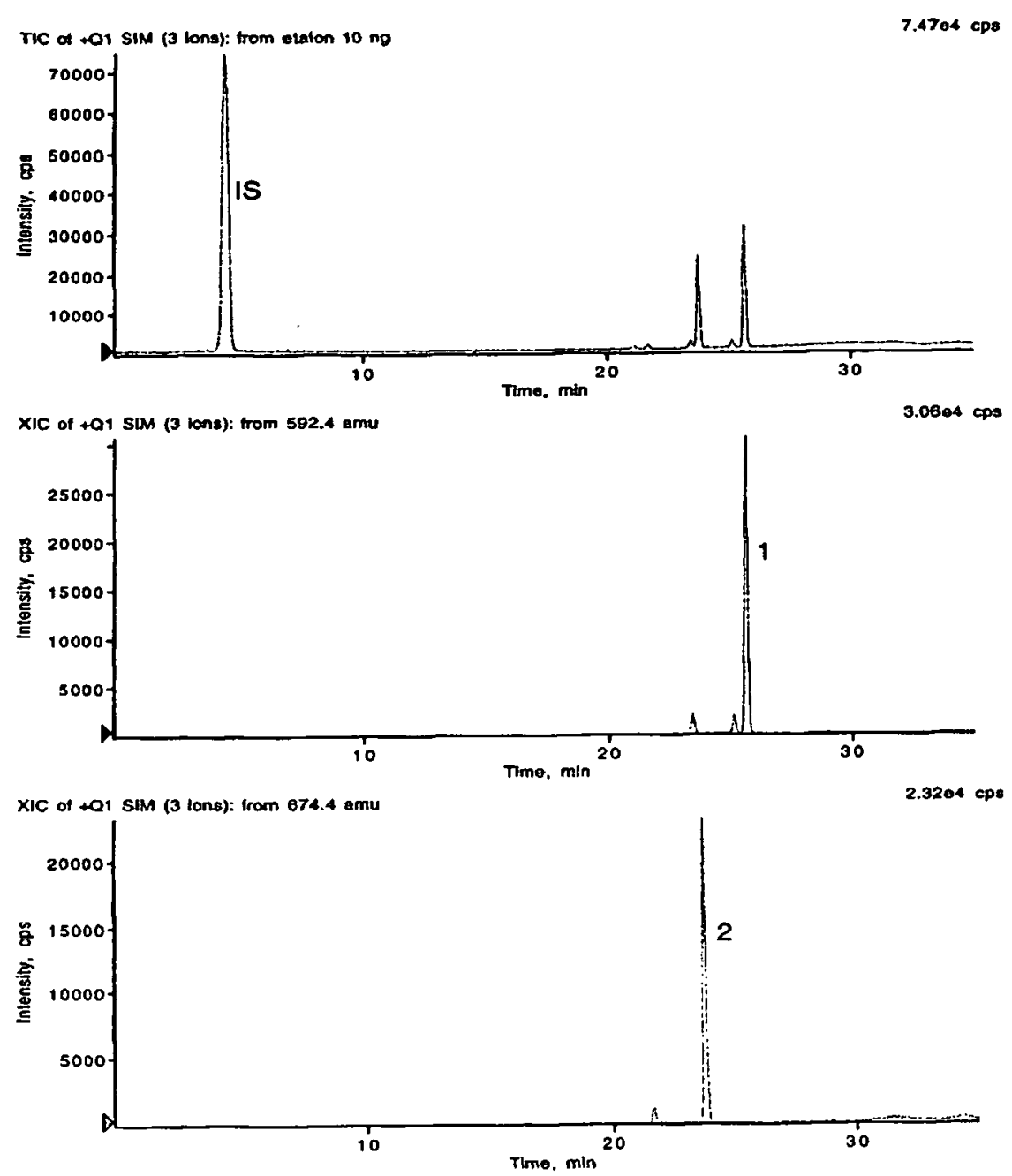

Figure 5 : Chromatogramme sur colonne ODS d'un extrait témoin avec ajout de $10 \mathrm{ng} / \mathrm{ml}$. Pics : 1 = cévadine $(\mathrm{m} / \mathrm{z}$ $592,4), 2$ = vératridine $(\mathrm{m} / \mathrm{z}, 674,4)$.

\section{CAS n 3 : Atropine}

Un homme d'âge non communiqué était retrouvé mort dans sa cellule de Maison d'Arrêt. La congestion polyviscérale retrouvée par le médecin légiste lors de l'autopsie orientait le décès sur une cause toxique. L'enquête n'a pas su déterminer s'il s'agissait d'un suicide ou d'un accident. La recherche de toxiques notamment végétaux réalisée un an après une première expertise où seule la présence d'alcool éthylique à $0,93 \mathrm{~g} / \mathrm{L}$ avait été mise en évidence, montrait l'ingestion d'atropine suivie d'un processus mortel très rapide puisque son absence de l'urine montrait que l'élimination n'avait pas commencé (tableau VII). La concentration plus faible dans le contenu gastrique que dans le sang indiquait que la résorption était sur la phase finale. Le décès pouvait ainsi être estimé entre une demi-heure et deux heures après la prise. Son origine est très vraisemblablement due à l'ingestion d'ampoules d'atropine (réservées à l'usage hospitalier en anesthésie-réanimation) déclarées volées à l'infirmerie de la Maison d'Arrêt.

Tableau VII : Cas 3 - Résultats d'analyse toxicologique.

\begin{tabular}{|l|l|}
\hline sang & éthanol $=0,71 \mathrm{~g} / 1$ \\
& atropine $=314 \mathrm{ng} / \mathrm{ml}$ \\
\hline urine & atropine $=$ non détectée \\
\hline contenu gastrique & atropine $=51 \mathrm{ng} / \mathrm{ml}$ \\
\hline
\end{tabular}

\section{CAS n ${ }^{\circ} 4$ : Colchicine}

Un homme de 55 ans était retrouvé mort à son domicile. L'autopsie mettait en évidence une intense congestion des viscères, pas de signes de violence ni de points d'injection. Des échantillons de sang et d'urine furent prélevés. Une première analyse révélait la présence de méprobamate (anxiolytique), de paracétamol (analgésique périphérique) et de phénobarbital (barbiturique sédatif et anti-convulsif) à taux thérapeutiques. Une deuxième analyse réalisée au laboratoire Toxlab 6 mois plus tard sur un sang prélevé depuis plus de deux ans révélait la présence d'acébutolol, un bêtabloquant principe actif du Sectral, prescrit par son médecin contre son hypertension artérielle. Elle révélait également la présence de morphine et de colchicine entrant dans la composition de Colchimax (opium, colchicine). Cet anti-goutteux n'apparaissait pas dans son traitement mais dans la liste des médicaments prescrits à sa femme. Les résultats sont regroupés dans le tableau VIII. La colchicine était présente à taux thérapeutique, cependant sa demi-vie de 20 minutes environ fait qu'elle est difficilement détectable en post-mortem (11). De plus en tant que poison mitotique elle entraîne une mort retardée. La présence de colchicine à ce taux $(0,79 \mathrm{ng} / \mathrm{ml})$ était donc en faveur d'une intoxication $(12,13)$. Contre toute attente, sa belle fille avouait finalement l'avoir empoisonné en écrasant des comprimés dans sa nourriture.

Tableau VIII : Cas 4 - Résultats d'analyse toxicologique dans le sang.

\begin{tabular}{|c|c|}
\hline $\begin{array}{l}\text { lre analyse } \\
(\mu \mathrm{g} / \mathrm{ml})\end{array}$ & $\begin{array}{c}\text { Méprobamate }=9 \\
\text { Paracétamol }=8,8 \\
\text { Phénobarbital }=17,5 \\
\text { Méthylphénobarbital }=8,5\end{array}$ \\
\hline 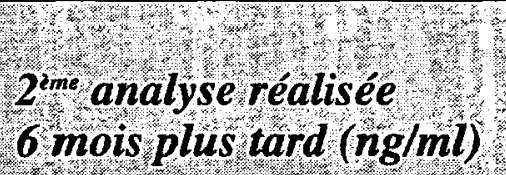 & $\begin{array}{c}\text { Acébutolol }=90 \\
\text { Morphine }=9 \\
\text { Colchicine }=0,79\end{array}$ \\
\hline
\end{tabular}

\section{CAS n 5 : Lobélie (Lobéline)}

Un jeune homme de 15 ans, d'origine martiniquaise était retrouvé mort dans son lit le matin, par un membre de sa famille. La congestion observée à l'autopsie était particulièrement notable sur le foie, la rate et les reins, mais aussi les poumons. Il était également observé des vomissures de sang. Des prélèvements de sang, d'urine, de viscères et de cheveux étaient effectués. Les analyses sanguines montraient la présence de lobéline $=20.8 \mathrm{ng} / \mathrm{ml}$, alcaloïde de la Lobélie, une plante naturelle d'Amérique du Nord, de Martinique et de Guadeloupe stimulant les mouvements respiratoires. 
Elle n'est actuellement plus utilisée en thérapeutique du fait de ses effets secondaires importants et de sa toxicité; cependant des jeunes gens, particulièrement aux Etats Unis, en fument pour ses effets euphorisants. La littérature ne rapporte pas de taux dans les fluides biologiques mais la présence significative de cet alcaloïde dans le sang peut être à l'origine d'une intoxication.

\section{Conclusion}

Il apparaît que la LC/MS et la LC/MS-MS sont des méthodes de choix pour la détermination de toxiques végétaux $(7,14)$. Cette méthode analytique appliquée à l'identification et au dosage des principaux alcaloïdes toxiques causes de décès, est la seule méthode de recherche large actuellement disponible permettant d'atteindre des limites de sensibilité de 1 à $0,1 \mathrm{ng} / \mathrm{ml}$ (voire moins). Elle a été appliquée avec succès dans différents cas médico-légaux. Elle devrait permettre à terme d'obtenir une évaluation plus juste du nombre d'intoxications par ces végétaux et des taux sanguins associés.

\section{Références}

1. Oztekin-Mat. A., Les intoxications d'origine végétale en Turquie. Ann. Pharm. Françaises. 1994 ; 52 : 260-265.

2. Krenzelok E.P., Jacobsen T.D., Plant exposures A national profile of the most common plant genera. Vet. Hum. Toxicol. $1997 ; 39: 248-249$.

3. FoukaridisG.N. , Muntigh G.L., Osuch E., Application of diode array detection for the identification of poisoning by traditional medicines. J. Ethnopharmacol. 1994 ; $41: 135-146$.

4. Hostege D.M. , Seiber J.N., Galey F.D., Rapid multiresidue screen for alkaloids in plant material and biological samples. J. Agric. Food Chem. 1995 ; 43 : 691-699.

5. Bruneton J., Plantes toxiques - Végétaux dangereux pour l'Homme et les animaux. Paris : Lavoisier, 1996.

6. Bruneton J., Pharmacognosie - Phytochimie, plantes médicinales. Paris : Lavoisier, 1993.

7. Gaillard Y., Pépin G., Review: Poisoning by plant material : review of human cases and analytical determination of main toxins by high performance liquid chromatography-(tandem) mass spectrometry. J. Chromatogr. B. 1999, $733: 181-229$.

8. Sticht G. , Kaferstein H., Staak M., Results of toxicological investigations of poisonings with atropine and scopolamine. Acta Med. Leg. Soc. 1989 ; 39 : 441-447.

9. Urich R.W. , Bowerman D.L. , Lewisky J.A., Pflug J.L., Datura stramonium : a fatal poisoning. J. Forensic Sci. $1982 ; 27: 948-954$.

10. Lange A., Toft P., Poisoning with nightshade, atropa belladonna. Laeger: Ugeskr. , 152, 1990, 1096.

11. Tracqui A., Kintz P., Ludes B., Rougé C., Douibi H., Mangin P., High-performance liquid chromatography coupled to ion spray mass spectrometry for the determination of colchicine at ppb levels in human biofluids. J. Chromatogr. B. 1996 ; 675 : 235-242.

12. Caplan Y.H., Orloff K.G., Tompson B.C., A fatal overdose with colchicine. J. Anal. Toxicol. $1980 ; 4$ : 153-155.

13. Kintz P., Jamey C., Tracqui A., Mangin P., Colchicine poisoning : report of a fatal case and presentation of an HPLC procedure for body fluid and tissue analyses. J. Anal. Toxicol. $1997 ; 21: 70-72$.

14. Tracqui A, Kintz P., Branche F., Ludes B., Confirmation of oleander poisoning by HPLC/MS. Int. J. Leg. Med. $1998 ; 111: 32-34$. 